\title{
Nutritional responses of cellular defence systems to different fats with or without phytochemical rich extracts: molecular markers of health status
}

\author{
J. E. Drew ${ }^{1}$, Garry Duthie ${ }^{1}$, A. J. Farquharson ${ }^{1}$ and Graham Horgan ${ }^{2}$ \\ ${ }^{1}$ Rowett Institute of Nutrition and Health, University of Aberdeen and ${ }^{2}$ Biomathematics and Statistics Scotland, \\ Greenburn Road, Bucksburn, Aberdeen, AB21 9SB, UK
}

Maintenance of homeostatic regulation in response to nutritional challenges is an indicator of health status. Cell defence systems, immunity, inflammation, redox regulation, metabolism and DNA repair are essential to maintain homeostatic regulation and are impacted by nutrition ${ }^{(1)}$. Human whole blood presents opportunities to assess cell defence system status in humans and identify food formulations to improve health status ${ }^{(1,2,3)}$. Males 21 to 60 years $(n=19)$ provided fasted baseline blood samples $(0 \mathrm{~h})$. Meal formulations, A (50\% unsaturated fat), B (50\% saturated fat), C (50\% unsaturated fat, $10 \mathrm{~g} / 100 \mathrm{~g}$ beetroot extract) and D (50\% saturated fat, $10 \mathrm{~g} / 100 \mathrm{~g}$ beetroot extract) were consumed by each subject on consecutive visits at least 1 week apart. Total RNA extracted from postprandial blood collected at $0 \mathrm{~h}, 1 \mathrm{~h}, 2 \mathrm{~h}, 4 \mathrm{~h}, 6 \mathrm{~h}$ and $24 \mathrm{~h}$ in PAXgene $($ blood RNA tubes (PreAnalytiX GmbH) and Blood RNA Kit (Qiagen, Crawley, UK) was analysed by Agilent Bioanalyser (Agilent Technologies, Bracknell, UK). Analysis of gene expression was then conducted using the GenomeLab System and an in-house designed multiplex assay, the hCellDSPlex. The hCellDSPlex incorporates cell defence system markers of immunity (CD54, CD62E, CEACAM5, CD69, CD106), redox (SOD2, NOS2, GPX1, HO1, NRF2, P22PHOX, GSS), inflammation (PTGS2, IL1B, IL10, IL6, TNFA, CRP), metabolism (SIRT1, $U C P 2, S O C S 3, C O X 6 C, C O Q 2)$ and DNA damage and repair (TP53, P16INK4A, GADD45A, APE1), red blood cell marker (EPB42) and reference genes (PPIA, PSMB6 and Kan $(r)$ ). Principal component analysis (PCA) revealed characteristic gene expression patterns within the group of study subjects (Figure 1). One-way ANOVA blocked for subject was applied, identifying cell defence system gene marker changes in gene expression profiles associated with differences in individual volunteers, meal formulation and time.

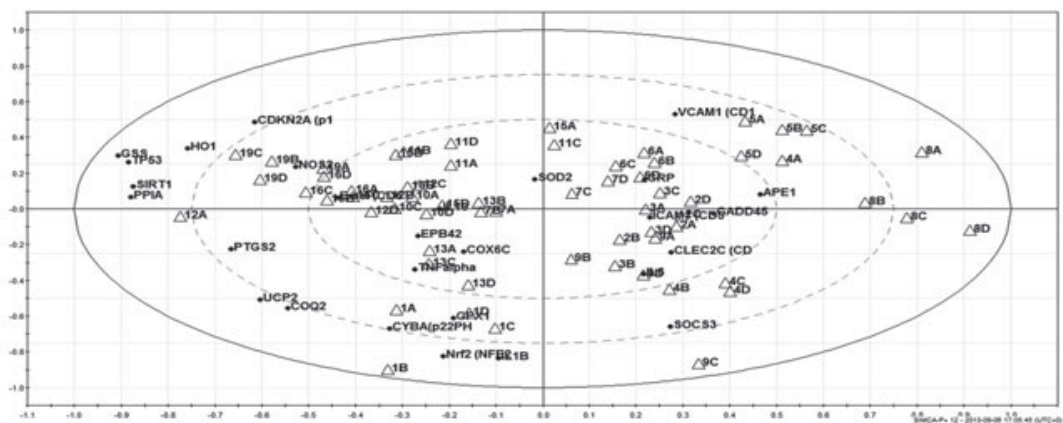

Fig. 1. PCA showing distinct gene expression patterns between study subjects (numbered triangles) tested at 0 hr on four different occasions (A, B, C and D). The genes contributing to characteristic profiles are indicated by $\bullet$ gene name.

This approach could be developed to monitor and survey the impact of diet-gene interactions to generate evidence for effective translation of research on food, drink and health.

Human Nutrition Unit and Genomics Unit staff (Rowett Institute of Nutrition Health). Funding: Scottish Government's Rural and Environment Science and Analytical Services Division.

1. Drew JE (2012) Advances in Nutrition 3, 1-7.

2. Drew JE, Mayer C-D, Farquharson AJ et al. (2011) J Mol Diagn 13, 233-242.

3. Drew JE \& Farquharson AJ (2012) Proc Nut Soc 71, E12. 